\title{
The therapeutic effect of autologous bone marrow mesenchymal stem cells to prevent the progress of chronic allograft nephropathy
}

\author{
Farhad Gholami $^{\mathbb{D}}$, Ali Ghasemi², Ahmad Reza Bahrami ${ }^{3}$, Hamid Reza Bidkhouri ${ }^{3}$, Hojat Naderi \\ Mishkin ${ }^{4}$, Masoud Pezeshki Rad ${ }^{5}$, Farzaneh Sharifipour ${ }^{6 *}$ \\ ${ }^{1}$ Department of Internal Medicine, Faculty of Medicine, Mazandaran University of Medical Sciences, Mazandaran, Iran \\ ${ }^{2}$ Department of Hematology and Pediatrics, Mashhad University of Medical Sciences, Mashhad, Iran \\ ${ }^{3}$ Department of Biology, Faculty of Sciences, Ferdowsi University Of Mashhad, Mashhad, Iran \\ ${ }^{4}$ Academic Center for Education, Culture and Research (ACECR), Stem Cell and Regenerative Medicine, Mashhad University of Medical \\ Sciences, Mashhad, Iran \\ ${ }^{5}$ Associate Professor of Radiology, Mashhad University of Medical Sciences, Mashhad, Iran \\ ${ }^{6}$ Division of Nephrology and Hypertension, Department of Internal Medicine, Mashhad University of Medical Sciences, Mashhad, Iran
}

\section{A R T I C L E I N F O}

Article Type:

Review

\section{Article History:}

Received: 7 February 2018

Accepted: 2 July 2018

Published online: 20 July 2018

\begin{abstract}
A B S T R A C T
Progenitor cells or mesenchymal stem cells are new cells, with renovation and regeneration ability and tissues repair. In recent years, administration of stem cells has been introduced to treat a variety of diseases including chronic allograft injury (CAI). It seems that stem cells can create a turning point in regenerative medicine through repair, replacement with damaged tissues or with their paracrine effects.
\end{abstract}

Keywords: Mesenchymal stem cells, End-stage renal disease, Chronic allograft nephropathy, Chronic allograft injury, Tubular atrophy, Interstitial fibrosis

Implication for health policy/practice/research/medical education:

Mesenchymal non-hematopoietic stem cells are undifferentiated progenitor bone marrow with the ability of renewal and reconstruction and healing of injured tissues.

Please cite this paper as: Gholami F, Ghasemi A, Bahrami AR, Bidkhouri HR, Naderi Mishkin H, Pezeshki Rad M, et al. The therapeutic effect of autologous bone marrow mesenchymal stem cells to prevent the progress of chronic allograft nephropathy.

J Renal Inj Prev. 2019;8(1):1-5. DOI: 10.15171/jrip.2019.01.

\section{Introduction}

Chronic allograft nephropathy/chronic allograft injury (CAN/CAI) is one of the most common causes of chronic renal failure and end stage in the transplanted kidney. This disorder with tubular atrophy and interstitial fibrosis (IF/TA) is expressed with no evidence of any other etiology at least 3 months after the graft (1). With improved immunosuppression and short-term survival of transplanted kidney, the CAI has become a major cause of late graft loss. Mesenchymal has the ability to transform to become kidney cells (2).

\section{Materials and Methods}

For this review, we used a variety of sources including PubMed/Medline, EBSCO, EMBASE, Web of Science, Google Scholar, Scopus and directory of open access journals (DOAJ). The search was conducted by using combinations of the following key words and/or their equivalents; mesenchymal stem cells, end-stage renal disease, chronic allograft nephropathy, chronic allograft injury, tubular atrophy and interstitial fibrosis.

Stem cells; history, definitions and types

Frideshtin for the first time in 1976 introduced stem cells as the primary source of osteoblast cells, adipose and chondrocytes. Mesenchymal stem cells (MSCs) are the non-hematopoietic undifferentiated progenitor cells, present in bone marrow cell as well as in the other cells. These cells have the efficiency of renovation and differentiation, mainly presented in bone marrow with the morphology of small spindle-shaped cells having large nucleus and organelles within the cell with round, objects and clear nuclei. Additionally, they have cellular processes that are short with long reticular fibers within the bone marrow surrounded by matrix (3). However, they are separated from the mesenchymal tissue thus named 
MSCs. Their shape is similar to fibroblasts, formerly they were known as fibroblast colony-forming units (FtCFU). MSCs that isolated from different tissues differ in proliferation and differentiation (4).

Apart from bone marrow, MSCs are also presented in other tissues such as adipose tissue, umbilical cord, blood, chorionic villous placenta, amniotic fluid, peripheral blood, fetal liver, and lung. The highest number of them can be seen in newborns. With increasing age, their proportion will decrease, while their number dropped by half, up to 80 years of age (5).

These cells are a group of multicellular organism's cells that retained their ability to self-renew and differentiate into other cell types as well (6).

Considering the transition of stem cells to the other cells and developmental processes, they are divided into three classes as follows:

1. Totipotent cells; these cells can become a full organ. The fertilized egg cells are in this category.

2. Pluripotent cells; they have the efficiency of transforming into any type of cell, but not a complete organism. Fetal cells have such ability.

3. Multipotent cells; they have only the ability to become specific cells such as blood cells.

4. Unipotential cells; these cells produce only one type of cell, while the self-renewal of stem distinguish them from other cells.

Based on the source of stem cells for transplantation, three types of cells are extracted and used.

1. Autologous cells; their origin is person's own cells. They are taken from peripheral blood, bone marrow or adipose tissue and other tissues.

2. Allogenic cells; the origin of these cells is from nonself-human tissue. Like the transplanted kidney from a relative and non-relative living or the death donor human receptor, they are grafted.

3. Xenogeneic cells; stem cells that are derived from nonhuman, from animal species, for example, embryonic cells from pigs to treat Parkinson's disease (7).

In fact, areas of interest within these cells are due to their unique clinical characteristics, benefits and fast developmental process.

Hematopoietic progenitor or stem cells have been known and used before, but progenitor cells or MSCs were the new cells that could also affect other tissues and even to be converted to them (5).

The differentiation of stem cells led to verify these elements as one of the important options in the treatment of incurable human diseases based on cell therapy or tissue engineering. The common feature of all types of human embryonic stem cells, amniotic fluid, umbilical cord and adult stem cells is unlimited self-renewal capacity and potential to differentiate into several cell lines. This capability plays the main role in the evolution of a mammal species throughout their life time (8).
Mesenchymal stem cells; special features

Non-hematopoietic stem cells have the potential and the ability to convert a variety of cells, including osteocytes, chondrocytes and myocytes, cardiomyocytes and even the neurons cells. These cells play an essential role in maintaining homeostasis of bone marrow and maturity arrangement of hematopoietic and non-hematopoietic cells (5). MSCs express a variety of cell surface antigens; however, none of them reveal any specific marker expressing their characteristics on these diverse population of cells, including differences in morphology, physiology and surface antigens. They express countless numbers of sticky molecules and extra-cellular matrix proteins, cytokines and growth factor receptors that are associated with the performance and interaction with bone marrow stromal cells (9). Those have been separated from bone marrow, include molecules that express CD44, CD 106 and, CD105 (SH2; Endoglin) Sca-1VCAM-1), CD166, CD29, CD73, (SH3 and SH4), CD90 (Thy-1), CD117, STRO-1 (3). However, the MSCs do not express the specific markers of the hematopoietic and the endothelial cells, including the CD14, CD34, CD45 CD11b, CD14, CD31, CD33, CD34, CD133, and CD45. This features mark differentiation of these cells from other the cells (3).

\section{Human adipose tissue as a source of MSCs}

Although bone marrow is thought to be the main source of isolation of these cells, significant reduction in the differentiation potential of cells during the aging process, and injuries to the person at the time of sampling limits the use of bone marrow as a source of the cell. Today, considerable attention has been paid to the adipose tissue as a source of mesenchymal cells. Adipose tissue is accessible easily with a minimum injury. Indeed efficiency of the results is hundred times more than MSCs. In recent years, scientists were able to produce adipose tissuederived MSCs in vitro (10). Various subcutaneous lipids are abundant storage, accessible as a source of adult stem cells. Almost all adult tissues contain mesenchymal cells such as bone marrow, peripheral blood, blood vessels, skeletal muscle, skin, liver and lipid tissue. Firstly, bone marrow stem cells have been considered as the best source for extracting MSCs. But since this is an invasive method of access to the source and possible damage to person during sampling, and, obtaining very limited amount of samples, marked decrease in differentiation potential of the cells during the process of aging, we tried to search for suitable resources. This approach, due to easier access to adipose tissue and minimal injury to the patient and also access to higher amount of sample is more acceptable. Accordingly, this method yielded higher material than what was found in the bone marrow $(11,12)$. Thereby, the use of adipose tissue as a source of cells to extract more is a convenient replacement of bone marrow MSCs. Human adipose tissue is a source of tissue for the isolation of MSCs because the tissue is obtained easily and usually 
available in adequate amounts in liposuction method and its cellular yield is higher than MSC source of tissue.

The stem cells obtained from human adipose tissue are the multi-efficiency cells able to express morphological and biochemical characteristics of adipocytes, osteoblasts, chondrocytes, under proper culture conditions. Also it was detected that they have the ability to generate sufficient number of cells for cell therapy and tissue engineering applications (13-15).

Specific characteristics of stem cells

- Undifferentiated cells/nonspecific

- Undifferentiated cells with the ability to transform into specialized cells and/or tissue-specific

- Self-renewal characteristics and tissue repair/replace damaged tissue cells caused by the pathogen $(3,16)$.

Stem cells, kidney failure and transplant

Stem cells, especially in acute kidney injury (AKI), and kidney transplanted were used.

MSCs after migration to damaged tissue and implanted in damaged tissue will release factors that increase the cell survival and the cell proliferation. They show antiinflammatory function and mediate immune response. Their anti-epidotic, anti-mutagenic, and proangiogenic properties have been shown faster recovery and tissue function in the kidney (17). These cells have been considered for therapeutic use due to the sum of these actions, combined with their availability and ability to spread out and development in in-vitro condition. Clinical and laboratory evidence has shown that treatment with these cells is safe and effective in many clinical studies $(18,19)$.

Cell therapy and tissue repair of damaged kidney

Squillaro et al reported tissue regeneration in acute renal failure with alternative replacement of these cells with tubular epithelial cells through the transformation of injected cells into the tubular cells (20).

This restorative effect, with the protective effect of mesenchymal cells in pre-clinical models such as renal ischemia, nephrotoxicity of cisplatin, myoglobin uricacute kidney failure and sepsis-related have been accessed (3). It is possible by infusion of autologous mesenchymal cells with subclinical rejection, or advanced IF/ TA.

It was shown that this treatment is better tolerated, improved the transplant rejection. Additionally the extension of opportunistic infection is reduced and a reduction in peripheral mononuclear blood cell proliferation was detected (21).

MSCs in acute renal failure

AKI is a clear cause of death. But not all patients with acute kidney dysfunction progress to chronic kidney disease (CKD). The human body features are a series of renovations that improve renal function following acute injury. Several studies have shown that stem cells can reduce kidney damage. Some data do not agree with the above mentioned point on the accompanying of MSCs with tubulogenesis (22).

Improvement in function and kidney structure has seen with MSCs. MSCs may participate in all reconstruction of endogenous capacity of kidney $(22,23)$.

\section{MSCs in chronic kidney disease}

MSCs may be used in CKD Patients. A recent investigation used the 5/6 nephrectomized mice as a model of CKD and tried to find out whether MSC improves this type of impaired kidney function or not? They found that protein and creatinine level significantly lowered in the MSC group after 30 days of therapy. Another study showed that blood pressure, urine protein, BUN and serum creatinine levels improved significantly 30 days after treatment. Recent studies indicate that MSCs are able to stimulate angiogenesis. MSCs transplantation produced angiogenesis and the formation of new blood vessels after 15 days.

The precise role of treatment with MSC is supported by its renoprotective role following acute damage. Additionally, the immunosuppressive and the immunemodulatory role of these cells have been identified. Their immunosuppressive ability following ischemic reperfusion injury (IRI), and cellular necrosis is due to pro-inflammatory waterfall which is caused by these cells. In the histologic investigation, loss of collagen and fibrosis in patients treated with MSCs were observed in the mice under study (24).

The fundamental role of MSCs is improving the renal microenvironment. In an initial cohort study, treatment with MSCS in eight patients with grade III and IV, graftversus-host disease (GVHD), recovery was noticed in $63 \%$ of patients. In 55 GVHD cases, treatment with MSCS infusion was administered intravenously. Around 30 patients showed a complete response and partial response was observed in 9 cases. Patients experienced a significant improvement in complete response compared to those who had not a complete response. These findings confirm the clinical application of the immunomodulatory effect of these cells (25).

Stem cells and transplanted kidneys

The use of MSCs in the treatment of kidney transplant rejection and chronic nephropathy due to toxic effects of cyclosporine (calcineurin nephrotoxicity) on allograft kidney has been conducted. In patients receiving stem cell no delayed graft function occurred. The use of stem cells in reducing delayed graft function is an effective, rapid and safe treatment (26). Studies have shown that autologous stem cell therapy in kidney transplant rejection allograft is safe and feasible (19). Injecting the MSCs in patients following kidney transplantation could greatly reduce the symptoms of graft rejection and graft survival. Injection of 
these cells had no complication for patients (18).

Direct injection of MSCs into the kidney arteries

The problem of inability to transfer many and effective mesenchymal living cells to the target tissues. Thus, favorable treatment in this route falls dramatically. This inefficient homing of MSCs is due to various factors, but mainly is attributed to the absence of surface receptors on the cells. Use of the arterial system and direct injection of cells into the feeding main artery and better effectiveness of this method has been shown to improve organ function in animal studies $(27,28)$. For the first time in the world, some researchers in Mashhad University of Medical Sciences used intra-arterial injection of MSCs in human to examine the effect of bone marrow MSCs in the treatment of chronic allograft injury/failure. This study was designed and carried out in phase 1 clinical trial to evaluate the safety and feasibility of the methods. Bone marrow stem cell nearly two - three hundred million cells injected through the renal artery of transplanted kidney. In none of the cases, the early and late onset reaction following to the injection at the first hour to the end of the first week and first and six month and after one year was happened. However, the effectiveness of this approach in improving performance in each of the patients according to age and duration of kidney transplantation and failure was differed.

\section{Conclusion}

Progenitor cells or MSCs are the new cells, with renovation and regeneration ability and tissues repair. Nephropathy, or chronic graft injury, is one of the most common causes of chronic renal failure in the transplanted kidney. In recent years, the use of stem cells has been introduced to treat a variety of diseases including chronic graft injury. It seems that stem cells can create a turning point in regenerative medicine through repair, replacement of damaged tissues by their distinctiveness efficiency or with their paracrine effects. It seems to be a new method to save patients prone to end-stage renal disease. There is a need for further research and development of new techniques such as intra-arterial injection of these cells and finding suitable conditions and continuing right and safe direction of these cells to the target tissue.

\section{Acknowledgements}

We would like to thank the director of the JAHAD Daneshgahi of Mashhad University and colleagues in the Department of Cellular and Molecular Research, Dr. Bidkhori, Montaserieh Hospital and directors of kidney transplant and bone marrow ward.

\section{Conflicts of interest}

The authors declared no competing interests.

\section{Ethical considerations}

Ethical issues (including plagiarism, data fabrication, double publication) have been completely observed by the authors.

Funding/Support

None.

\section{References}

1. Riella LV, Djamali A, Pascual J. Chronic allograft injury: Mechanisms and potential treatment targets. Transplant Rev (Orlando). 2017;31:1-9. doi: 10.1016/j.trre.2016.10.005.

2. Nankivell BJ, Chapman JR. Chronic allograft nephropathy: current concepts and future directions. Transplantation. 2006;81:643-54. doi:10.1097/01.tp.0000190423.82154.01.

3. Bobis S, Jarocha D, Majka M. Mesenchymal stem cells: characteristics and clinical applications. Folia Histochem Cytobiol. 2006;44:215-30.

4. Bianco P. "Mesenchymal" stem cells. Annu Rev Cell Dev Biol. 2014;30:677-704. doi: 10.1146/annurevcellbio-100913-013132.

5. Undale AH, Westendorf JJ, Yaszemski MJ, Khosla S. Mesenchymal stem cells for bone repair and metabolic bone diseases. Mayo Clin Proc. 2009;84:893-902. doi: 10.1016/ S0025-6196(11)60506-5.

6. Singh S, Mackill DJ, Ismail AM. Physiological basis of tolerance to complete submergence in rice involves genetic factors in addition to the SUB1 gene. AoB Plants. 2014;6. doi: 10.1093/aobpla/plu060.

7. Temple S. The development of neural stem cells. Nature. 2001;414:112-7.

8. Ding DC, Chang YH, Shyu WC, Lin SZ. Human umbilical cord mesenchymal stem cells: a new era for stem cell therapy. Cell Transplant. 2015;24:339-47. doi: $10.3727 / 096368915$ X686841.

9. Shi Y, Hu G, Su J, Li W, Chen Q, Shou P, et al. Mesenchymal stem cells: a new strategy for immunosuppression and tissue repair. Cell Res. 2010;20:510-8. doi: 10.1038/cr.2010.44.

10. Rodriguez AM, Elabd C, Amri EZ, Ailhaud G, Dani C. The human adipose tissue is a source of multipotent stem cells. Biochimie. 2005;87:125-8. doi:10.1016/j. biochi.2004.11.007.

11. Bacakova L, Zarubova J, Travnickova M, Musilkova J, Pajorova J, Slepicka P, et al. Stem cells: their source, potency and use in regenerative therapies with focus on adiposederived stem cells - a review. Biotechnol Adv. 2018;36:11111126. doi: 10.1016/j.biotechadv.2018.03.011.

12. Zuk PA, Zhu M, Ashjian P, De Ugarte DA, Huang JI, Mizuno $\mathrm{H}$, et al. Human adipose tissue is a source of multipotent stem cells. Mol Biol Cell. 2002;13:4279-95. doi:10.1091/ mbc.e02-02-0105.

13. Perico N, Casiraghi F, Introna M, Gotti E, Todeschini M, Cavinato RA, et al. Autologous mesenchymal stromal cells and kidney transplantation: a pilot study of safety and clinical feasibility. Clin J Am Soc Nephrol. 2011;6:412-22. doi: $10.2215 /$ CJN.04950610.

14. Elahi M, Kabir Salmani M, Shiravi A. Extraction of human mesenchymal stem cells derived from adipose tissue using various enzymes. Research Med. 2012;35:200-208.

15. Gimble JM, Katz AJ, Bunnell BA. Adipose-derived stem cells for regenerative medicine. Circ Res. 2007;100:1249-60. doi: 10.1161/01.RES.0000265074.83288.09.

16. Shi M, Liu ZW, Wang FS. Immunomodulatory properties and therapeutic application of mesenchymal stem cells. 
Clin Exp Immunol. 2011;164:1-8. doi: 10.1111/j.13652249.2011.04327.x.

17. Eirin A, Lerman LO. Mesenchymal stem cell treatment for chronic renal failure. Stem Cell Res Ther. 2014;5:83. doi: $10.1186 /$ scrt 472 .

18. Reinders ME, de Fijter JW, Roelofs H, Bajema IM, de Vries DK, Schaapherder AF, et al. Autologous bone marrowderived mesenchymal stromal cells for the treatment of allograft rejection after renal transplantation: results of a phase I study. Stem Cells Transl Med. 2013;2:107-11. doi: $10.5966 / \mathrm{sctm} .2012-0114$.

19. Casiraghi F, Remuzzi G, Perico N. Mesenchymal stromal cells to promote kidney transplantation tolerance. Curr Opin Organ Transplant. 2014;19:47-53. doi: 10.1097/ MOT.0000000000000035.

20. Squillaro T, Peluso G, Galderisi U. Clinical Trials With Mesenchymal Stem Cells: An Update. Cell Transplant. 2016;25:829-48. doi: 10.3727/096368915X689622.

21. Rosenberg ME. Cell-based therapies in kidney disease. Kidney Int Suppl (2011). 2013;3:364-367. doi: 10.1038/ kisup.2011.16.

22. Tumlin J, Wali R, Williams W, Murray P, Tolwani AJ, Vinnikova AK, et al. Efficacy and safety of renal tubule cell therapy for acute renal failure. J Am Soc Nephrol. 2008;19:1034-40. doi: 10.1681/ASN.2007080895.

23. Herrera MB, Bussolati B, Bruno S, Fonsato V, Romanazzi
GM, Camussi G. Mesenchymal stem cells contribute to the renal repair of acute tubular epithelial injury. Int J Mol Med. 2004;14:1035-41.

24. Zhuo W, Liao L, Fu Y, Xu T, Wu W, Yang S, et al. Efficiency of endovenous versus arterial administration of mesenchymal stem cells for ischemia-reperfusion-induced renal dysfunction in rats. Transplant Proc. 2013;45:503-10. doi: $\quad 10.1016 / j . t r a n s p r o c e e d .2012 .07 .162$.

25. Torres Crigna A, Daniele C, Gamez C, Medina Balbuena S, Pastene DO, Nardozi D, et al. Stem/Stromal Cells for Treatment of Kidney Injuries With Focus on Preclinical Models. Front Med (Lausanne). 2018 Jun 15; 5:179. doi: 10.3389/fmed.2018.00179.

26. Vanikar AV, Trivedi HL, Kumar A, Gopal SC, Kute VB. Mesenchymal stem cells and transplant tolerance. Nephrology (Carlton). 2014;19:369-74. doi: 10.1111/ nep. 12258

27. Tan J, Wu W, Xu X, Liao L, Zheng F, Messinger S, et al. Induction therapy with autologous mesenchymal stem cells in living-related kidney transplants: a randomized controlled trial. JAMA. 2012;307:1169-77. doi: 10.1001/ jama.2012.316.

28. Humphreys BD, Bonventre JV. Mesenchymal stem cells in acute kidney injury. Annu Rev Med. 2008;59:311-25. doi: 10.1146/annurev.med.59.061506.154239.

Copyright (c) 2019 The Author(s); Published by Nickan Research Institute. This is an open-access article distributed under the terms of the Creative Commons Attribution License (http://creativecommons.org/licenses/by/4.0), which permits unrestricted use, distribution, and reproduction in any medium, provided the original work is properly cited. 